\title{
Efeito da solarização e da adubação sobre artrópodes em solo cultivado com alface
}

\author{
Marlene G da Silva ${ }^{1}$; Charles M de Oliveira ${ }^{2}$; Ana Maria R Junqueira ${ }^{1}$ \\ ${ }^{1}$ UnB, C. Postal 4.508, 70910-970 Brasília-DF; 2Embrapa Cerrados, C. Postal 08223, 73310-970 Planaltina-DF; lelenegs@yahoo.com.br; \\ charles@cpac.embrapa.br; anamaria@unb.br
}

\section{RESUMO}

Avaliou-se o efeito da solarização e da adubação química e orgânica na comunidade de artrópodes de solo na cultura da alface, cv. Verônica. Foram conduzidos dois experimentos, em blocos ao acaso, um com solarização e o outro sem solarização, em casa de vegetação, ambos com cinco tratamentos: adubação orgânica; nitrogênio na forma amoniacal $\left(\mathrm{NH}_{4}\right)$; adubação com NPK; adubação orgânica + NPK; testemunha (sem adubação). No experimento solarizado, o solo foi coberto durante 132 dias com plástico transparente. Os artrópodes foram coletados por meio de armadilhas tipo alçapão em três épocas (antes da implantação do experimento, após a solarização e após a colheita). Foram utilizados índices faunísticos e de diversidade em cada experimento, tratamento e épocas. Os principais grupos coletados foram: Collembola (82,8\%), Acari (7,1\%), Hymenoptera $(6,1 \%)$ Coleoptera $(1,3 \%)$ e outros $(2,7 \%)$. A classe Collembola foi a mais abundante, mesmo na colheita, quando a subordem Acari aumentou substancialmente. As espécies de Collembola predominantes foram: Proisotoma tenella (Reuter) (34,3\%); Seira atrolutea (Arlé) (29\%); Folsomides centralis (Denis) (7,2\%); Isotomurus sp. $161(2,4 \%)$ e Sminthurides sp. 98 (1,6\%). Observou-se que a diversidade e abundância da comunidade de artrópodes não foi influenciada pela solarização ou adubação.

Palavras-chave: Lactuca sativa L., fauna; fertilizante, estufa, tratamento térmico, energia solar.

\begin{abstract}
Solarization, organic and chemical fertilization combined effects on arthropods community in soil cultivated with lettuce

To evaluate the effect of solarization, chemical and organic fertilization on soil arthropods community in lettuce, two blocks plots trials were carried out in Brasília, Brazil. One research was conducted with solarization and the other without solarization in a soil cultivated with lettuce, cv Veronica, under protected cultivation. Each trial consisted of five fertilization treatments: $\mathrm{T} 1=$ organic fertilization; $\mathrm{T} 2=$ amoniacal nitrogen (ammonium sulphate); $\mathrm{T} 3=$ chemical fertilization; $\mathrm{T} 4=$ organic and chemical fertilization; $\mathrm{T} 5=$ control (without fertilization). In the solarized trial, the soil was covered for 132 days with transparent plastic. The arthropods were collected by pitfalls at three moments: 1) before solarization, 2) after solarization and 3) at harvest. Faunistic indexes were calculated in each trial, treatment and moments. The main collected groups were: Collembola (82.8\%), Acari (7.1\%), Hymenoptera (6.1\%), Coleoptera $(1.3 \%)$ and others $(2.7 \%)$. Collembola class represented the largest proportion of the arthropods, even at harvest when the sub-order Acari substantially increased. The predominant species of Collembola were: Proisotoma tenella (Reuter) (34.3\%); Seira atrolutea (Arlé) (29\%); Folsomides centralis (Denis) (7.2\%); Isotomurus sp.161 (2.4\%) and Sminthurides sp. 98 (1.6\%). Arthropods community was not affected by solarization or fertilization.
\end{abstract}

Keywords: Lactuca sativa L., fauna, fertilizer, greenhouse, thermal treatment, solar energy.

\section{(Recebido para publicação em 8 de outubro de 2008; aceito em 4 de novembro de 2009)} (Received in October 8, 2008; accepted in November 4, 2009)

\begin{abstract}
$\mathrm{C}$ omunidades ou mesmo grupos específicos de organismos podem ser usados como indicadores biológicos da qualidade do solo (Duarte, 2004). Nesse contexto, os artrópodes podem atuar como indicadores da deterioração do solo antes que as mudanças em suas propriedades ou na vegetação sejam evidentes (Greenslade \& Greenslade, 1983). Variáveis como abundância e diversidade de espécies de artrópodes têm sido utilizadas para a avaliação do impacto de tecnologias recentes de manejo de pragas (Eckert et al., 2006; Rose \& Dively, 2007) e o estudo das comunidades de artrópodes vem sendo utilizado como uma das técnicas para se avaliar mudanças no ambiente.
\end{abstract}

Os microartrópodes do solo apresentam enorme abundância e diversidade e podem funcionar como indicadores dos impactos antropogênicos (Greenslade \& Greenslade, 1983; Hasegawa, 2001; Duarte, 2004; Nakamura et al., 2007). Estudos sobre a comunidade de artrópodes em sistemas agrícolas são escassos, sobretudo no Brasil. Em alguns estudos realizados no Brasil Central observouse que o uso do solo para atividades agropastoris reduz a abundância de artrópodes do solo em relação à áreas de Cerrado virgem (Oliveira et al., 2002; Oliveira et al., 2006).

Os microartrópodes do solo desempenham papel importante em processos de decomposição e mineralização da matéria orgânica, principalmente ácaros e colêmbolos, os quais normalmente constituem a maior parte da artropodofauna edáfica (Oliveira et al., 2006; Lins et al., 2007; Badji et al., 2007). Esses organismos se alimentam de fungos de solo e restos vegetais, favorecendo a decomposição microbiana desses últimos (Spain \& Hutson, 1983; Assad, 1997; Hasegawa 2001; Zeppelini Filho \& Belini, 2004).

Em sistemas agrícolas, a adição de fertilizantes orgânicos pode favorecer a comunidade de artrópodes, por aumentar a biomassa vegetal e promover maior retorno de matéria orgânica ao solo (Leroy et al., 2007). Por outro lado, a aplicação de pesticidas pode causar impactos 
negativos nessas comunidades (Lins et al., 2007; Ferraro et al., 2007), e no meio ambiente, razão pela qual técnicas alternativas, como a solarização do solo vem sendo estudada para controle de pragas, patógenos e plantas daninhas, como por exemplo em cultivos de hortaliças. Por essa técnica, o solo umedecido é coberto com plástico transparente ficando exposto à luz do sol, durante os meses de altas radiações (Ghini et al., 2003). A solarização associada à adubação favorece o controle de pragas do solo, principalmente nematóides fitoparasitas (Hasing et al., 2004).

No Brasil praticamente inexistem informações sobre os efeitos da solarização e da adubação do solo na comunidade de artrópodes. $\mathrm{O}$ objetivo deste trabalho foi avaliar o efeito da solarização combinada à adubação química e orgânica na comunidade de artrópodes em solo cultivado com alface sob cultivo protegido, com base nas variáveis abundância e diversidade de espécies.

\section{MATERIAL E MÉTODOS}

O trabalho foi conduzido em casa de vegetação em estação experimental da UnB, em Brasília (latitude 15 56'00” S; longitude: $57^{\circ} 56^{\prime} 00^{\prime \prime} \mathrm{W}$; altitude 1080 $\mathrm{m}$, clima tropical de altitude). O solo é do tipo Latossolo Vermelho Amarelo, textura argilosa. Foi utilizada a cultivar de alface Verônica.

A casa de vegetação de $70 \mathrm{~m}^{2}$ foi dividida em duas partes, onde foram implantados dois experimentos, um com solarização e outro sem solarização. Em cada experimento, nas parcelas de $1,0 \times 1,8 \mathrm{~m}$, foram implantados os seguintes tratamentos de adubação: adubação orgânica (T1); nitrogênio na forma amoniacal (sulfato de amônio) (T2); adubação com NPK, de acordo com a análise de solo (T3); adubação orgânica mais NPK (T4); testemunha (sem adubação) (T5).

Em cada um dos experimentos utilizou-se um delineamento em blocos ao acaso com três repetições em parcelas subdivididas (cinco adubações $\mathrm{x}$ três épocas). Foram consideradas como parcelas, em cada experimento, os cinco tratamentos de adubação e como sub-parcelas as três épocas de coleta de amostras.

Antes da colocação do plástico em 01/09/2004, o solo foi preparado por aração e gradagem e irrigado até atingir a capacidade de campo. Os tratamentos $\mathrm{T} 1, \mathrm{~T} 2$, e T4 foram adubados respectivamente com $5,4 \mathrm{~kg}$ de esterco bovino, $135 \mathrm{~g}$ de sulfato de amônio e 5,4 kg de esterco bovino. Visando evitar o efeito de borda, a solarização foi realizada durante 132 dias (24 de setembro de 2004 a 2 fevereiro de 2005), utilizandose uma lona plástica transparente de polietileno, de $150 \mu \mathrm{m}$ de espessura. Em 10/02/2005, após a retirada do plástico, foi realizada a adubação das parcelas, em pré-plantio com as respectivas doses: $\mathrm{T} 1=5,4 \mathrm{~kg}$ de esterco bovino; $\mathrm{T} 2=60 \mathrm{~g}$ de superfosfato simples $\left(10,8 \mathrm{~g} \mathrm{P}_{2} \mathrm{O}_{5}\right) \mathrm{e}$ $12 \mathrm{~g}$ de cloreto de potássio $\left(7,2 \mathrm{~g} \mathrm{~K}_{2} \mathrm{O}\right)$; $\mathrm{T} 3=27 \mathrm{~g}$ de sulfato de amônio $(5,4 \mathrm{~g}$ de $\mathrm{N}), 60 \mathrm{~g}$ de superfosfato simples $(10,8$ $\mathrm{g}$ de $\mathrm{P}_{2} \mathrm{O}_{5}$ ) e $12 \mathrm{~g}$ de cloreto de potássio $\left(7,2 \mathrm{~g} \mathrm{~K}_{2} \mathrm{O}\right) ; \mathrm{T} 4=27 \mathrm{~g}$ de sulfato de amônio $(5,4 \mathrm{~g}$ de $\mathrm{N}), 60 \mathrm{~g}$ de superfosfato simples $\left(10,8 \mathrm{~g}\right.$ de $\left.\mathrm{P}_{2} \mathrm{O}_{5}\right)$ e $12 \mathrm{~g}$ de cloreto de potássio $\left(7,2 \mathrm{~g} \mathrm{~K}_{2} \mathrm{O}\right)$ e $5,4 \mathrm{~kg}$ de esterco bovino; T5 = sem adubação. As mudas de alface com 20 dias foram transplantadas em 15/02/2005, com espaçamento de $0,25 \mathrm{~m}$ entre linhas e entre plantas, totalizando 21 plantas por parcela. As adubações de cobertura em cada tratamento foram realizadas em 28/02/2005 (aos 15 dias) e em 18/03/2005 (aos 30 dias), aplicando-se em cada tratamento as seguintes doses: $\mathrm{T} 1=500 \mathrm{~g}$ de esterco bovino; $\mathrm{T} 2=72 \mathrm{~g}$ de sulfato de amônio e $12 \mathrm{~g}$ de cloreto de potássio; $\mathrm{T} 3=72 \mathrm{~g}$ de sulfato de amônio e 12 g cloreto de potássio; T4= $6 \mathrm{~g}$ de cloreto de potássio, $36 \mathrm{~g}$ de sulfato de amônio e $250 \mathrm{~g}$ de esterco bovino; T5= sem adubação.

Para coleta dos artrópodes foram instaladas no centro de cada parcela armadilhas de solo tipo alçapão (pit fall), constituídas por recipientes cilindros de plástico de $9 \mathrm{~cm}$ de diâmetro e $10 \mathrm{~cm}$ de altura, fechado no fundo e aberto em sua porção superior onde foi colocado um funil com abertura central de $5 \mathrm{~cm}$ de diâmetro. Foram enterradas ao nível do solo, e continham em seu interior $200 \mathrm{~mL}$ de solução de sulfato de cobre a $1 \%$. As armadilhas foram instaladas em três épocas: $1^{\mathrm{a}}$ ) antes da solarização $\left.(27 / 08 / 2004) ; 2^{\mathrm{a}}\right)$ logo após a solarização $(02 / 02 / 2005)$; e $\left.3^{\text {a }}\right)$ no final do ciclo da alface $(30 / 03 / 2005)$ e permaneceram por cinco dias consecutivos na área de estudo, sendo então recolhidas e levadas para laboratório.

No laboratório, os insetos e outros artrópodes foram transferidos para recipientes contendo álcool $70 \%$ e posteriormente foram separados e contados. A identificação foi realizada em nível de gênero e espécie, com base na literatura, por comparação com exemplares de museus entomológicos ou enviados a especialistas. Quando a identificação específica não foi possível, os espécimes foram classificados em nível de morfoespécies com o intuito de englobar toda a comunidade coletada.

Foi realizada uma análise faunística visando selecionar as espécies predominantes, isto é, aquelas que obtiveram maiores índices faunísticos, calculados pelas seguintes medidas da fauna: abundância, freqüência, constância e dominância. Para estudo da diversidade da comunidade de artrópodes, foram calculados: Índice de riqueza de Margalef $(\alpha)$, índice de diversidade de ShannonWiener ( $\left.\mathrm{H}^{\prime}\right)$, índice de Equitabilidade (E). Os índices foram calculados com base no aplicativo ANAFAU, desenvolvido pelo setor de entomologia da ESALQ/USP.

Para avaliação do efeito da solarização na comunidade de artrópodes, a análise faunística e de diversidade foi procedida com os dados totais de cada experimento. Para avaliação da comunidade de artrópodes em função das épocas e da solarização, utilizaram-se os dados totais de cada época em cada experimento. O efeito combinado da solarização e tratamentos de adubação na comunidade de artrópodes foi avaliado pelos dados totais de cada tratamento em cada experimento. E finalmente, o efeito combinado de solarização, épocas e adubações foi avaliado pelos dados de cada tratamento de adubação, em cada época e em cada experimento. Os dados de abundância, número de espécies e os índices de diversidade (índice de riqueza de Margalef $(\alpha)$, índice de diversidade de Shannon-Wiener (H') e índice de Equitabilidade (E)) foram comparados por meio do intervalo de confiança pelo 
teste $\mathrm{t}$ ao nível de 0,05 de probabilidade conforme Pimentel-Gomes (2000).

\section{RESULTADOS E DISCUSSÃO}

Foram coletados nos dois experimentos (com e sem solarização) nos cinco tratamentos e nas três épocas um total de 18.921 espécimes, distribuídos em seis classes, 16 ordens, 98 famílias e 225 espécies (Tabelas 1 e 2).

Os principais grupos coletados foram a classe Collembola (82,8 \%); a sub classe Acari (7,1\%); as ordens da classe Insecta, Hymenoptera $(6,1 \%)$ e Coleoptera (1,3\%); e outros $(2,7 \%)$ (o somatório das ordens Isoptera, Diptera, Hemiptera, Lepidoptera, Orthoptera, Psocoptera, Thysanoptera, Dermaptera, Araneae, Isopoda e as classes Diplopoda e Chilopoda) (Figura 1). Observou-se grande semelhança entre os grupos de artrópodes nos dois experimentos (Figura 1).

Colêmbolos e ácaros constituem a maior parte da mesofauna edáfica nos solos do Cerrado Brasileiro (Assad, 1997). Essa tendência também foi observada na Austrália (Spain \& Hutson, 1983), Guiana Francesa (Kounda-Kiki et al., 2004), Tailândia (Wiwatwitaya \& Takeda, 2005), Bélgica (Leroy et al., 2007) e Argentina (Ferraro et al., 2007). Wiwatwitaya \& Takeda (2005) encontraram em floresta maior proporção de ácaros $(75,3 \%)$ do que de colêmbolos (16,1\%). No Brasil, em áreas de mata de araucária (Duarte, 2004) e em áreas de Cerrado (Oliveira et al., 2006), tanto em solo sob vegetação nativa quanto em solo cultivado, observou-se predominância de ácaros. A maior abundância de colêmbolos encontrada no presente estudo, pode ser explicada pelo uso de armadilhas do tipo alçapão (pit fall), que coletam principalmente artrópodes epígeos (que vivem basicamente na superfície do solo) como são a maioria dos colêmbolos, enquanto que outros métodos de coleta, como o funil de Berlese-Tullgren utilizados por Duarte (2004) e Oliveira et al. (2006), coletam em sua maioria artrópodes que vivem no interior do solo, onde os colêmbolos são menos freqüentes.

As espécies mais abundantes pertenceram à classe Collembola: Proisotoma tenella (Reuter) (34,3\%); Seira atrolutea (Arlé) (29\%); Folsomides centralis (Denis) (7,2\%); Isotomurus sp. (2,4\%) e Sminthurides sp. (1,6\%). Em florestas da Tailândia, espécies de colêmbolos do gênero Proisotoma e Folsomides foram as mais abundantes (Wiwatwitaya \& Takeda, 2005). Além destas espécies,

Tabela 1. Total de espécimes coletados, número de espécies, índice de riqueza de Margalef $(\alpha)$, índice de diversidade de Shannom-Wiener (H') e equitabilidade (E) em armadilha tipo alçapão nos experimentos com e sem solarização para as três épocas e nos cinco tratamentos de adubação (total of collected specimens, number of species, Margalef richness index ( $\alpha$ ), Shannom-Wiener diversity index (H') and equitability (E) in pitfalls in solarized and unsolarized experiments in three periods and five fertilization treatments). Brasília, UnB, 2005.

\begin{tabular}{|c|c|c|c|c|c|}
\hline Épocas/adubações & Total coletado & $N^{\circ}$ de espécies & $\alpha$ & $\mathbf{H}^{\prime}$ & $\mathbf{E}$ \\
\hline \multicolumn{6}{|l|}{ Entre experimentos } \\
\hline Com solarização & $9375 \mathrm{aA}$ & $165 \mathrm{aA}$ & $17,93 \mathrm{aA}$ & $2,31 \mathrm{aA}$ & $0,34 \mathrm{aA}$ \\
\hline Sem solarização & $9546 \mathrm{aA}$ & $175 \mathrm{aA}$ & $18,99 \mathrm{aA}$ & $2,48 \mathrm{aA}$ & $0,36 \mathrm{aA}$ \\
\hline \multicolumn{6}{|l|}{ Com solarização } \\
\hline 1 & $2964 b A$ & $52 \mathrm{bA}$ & $6,38 \mathrm{cA}$ & $1,60 \mathrm{cA}$ & $0,41 \mathrm{bA}$ \\
\hline 2 & $5322 \mathrm{aA}$ & $90 \mathrm{aA}$ & $10,37 \mathrm{bA}$ & $1,93 \mathrm{bA}$ & $0,43 \mathrm{bA}$ \\
\hline 3 & $1089 \mathrm{cA}$ & $106 \mathrm{aA}$ & $15,02 \mathrm{aA}$ & $3,46 \mathrm{aA}$ & $0,74 \mathrm{aA}$ \\
\hline Orgânica & $2145 \mathrm{aA}$ & $91 \mathrm{aA}$ & $11,73 \mathrm{aA}$ & $2,33 \mathrm{aA}$ & $0,52 \mathrm{aA}$ \\
\hline $\mathrm{N}-\mathrm{NH}_{4}$ & $1622 \mathrm{aA}$ & $75 \mathrm{aA}$ & $10,01 \mathrm{aA}$ & $2,23 \mathrm{aA}$ & $0,52 \mathrm{aA}$ \\
\hline NPK & $2093 \mathrm{aA}$ & $70 \mathrm{aA}$ & $9,02 \mathrm{aA}$ & $1,97 \mathrm{aA}$ & $0,46 \mathrm{aA}$ \\
\hline Org. + NPK & $1609 \mathrm{aA}$ & $101 \mathrm{aA}$ & $13,54 \mathrm{aA}$ & $2,47 \mathrm{aA}$ & $0,53 \mathrm{aA}$ \\
\hline Testemunha & $1906 \mathrm{aA}$ & $78 \mathrm{aA}$ & $10,19 \mathrm{aA}$ & $2,19 \mathrm{aA}$ & $0,50 \mathrm{aA}$ \\
\hline \multicolumn{6}{|l|}{ Sem solarização } \\
\hline 1 & $2475 \mathrm{bA}$ & $44 \mathrm{bA}$ & $5,50 \mathrm{bA}$ & $1,65 \mathrm{cA}$ & $0,44 \mathrm{bA}$ \\
\hline 2 & $5367 \mathrm{aA}$ & $105 \mathrm{aA}$ & $12,11 \mathrm{aA}$ & $2,07 \mathrm{bA}$ & $0,44 \mathrm{bA}$ \\
\hline 3 & $1704 \mathrm{bA}$ & $112 \mathrm{aA}$ & $14,92 \mathrm{aA}$ & $3,23 \mathrm{aA}$ & $0,69 \mathrm{aA}$ \\
\hline Orgânica & $2118 \mathrm{aA}$ & $102 \mathrm{aA}$ & $13,19 \mathrm{aA}$ & $2,38 \mathrm{aA}$ & $0,52 \mathrm{aA}$ \\
\hline $\mathrm{N}-\mathrm{NH}_{4}$ & $1961 \mathrm{aA}$ & $87 \mathrm{aA}$ & $11,34 \mathrm{aA}$ & $2,23 \mathrm{aA}$ & $0,50 \mathrm{aA}$ \\
\hline $\mathrm{NPK}$ & $1721 \mathrm{aA}$ & $73 \mathrm{aA}$ & $9,66 \mathrm{aA}$ & $2,13 \mathrm{aA}$ & $0,50 \mathrm{aA}$ \\
\hline Org. + NPK & $2035 \mathrm{aA}$ & $106 \mathrm{aA}$ & $13,78 \mathrm{aA}$ & $2,77 \mathrm{aA}$ & $0,59 \mathrm{aA}$ \\
\hline Testemunha & $1711 \mathrm{aA}$ & $86 a A$ & $11,42 \mathrm{aA}$ & $2,20 \mathrm{aA}$ & $0,49 \mathrm{aA}$ \\
\hline
\end{tabular}

Médias de épocas e de adubações dentro de cada experimento seguidas de mesma letra minúscula na coluna e entre épocas e adubações dos dois experimentos seguidas de letra maiúscula na coluna, não diferem pelo intervalo de confiança do teste $\mathrm{t}$ a 0,05 de probabilidade. (Periods or fertilization averages folowed by same small letter in the column and periods and fertilization averages folowed by same capital letter in the column did not differ from each other by the $t$ test at $5 \%$ probability). 


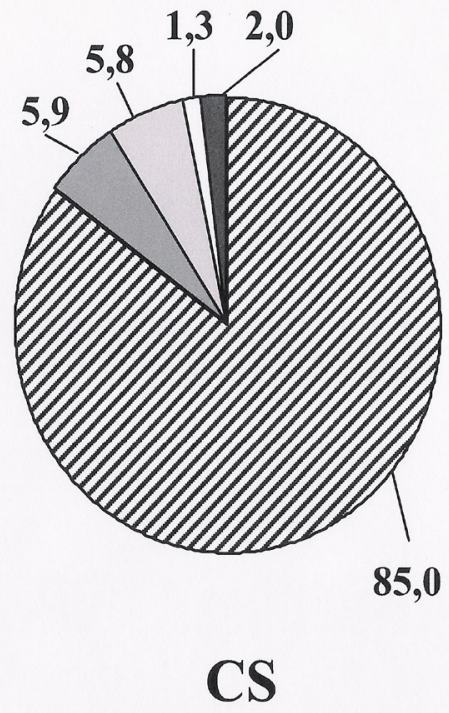

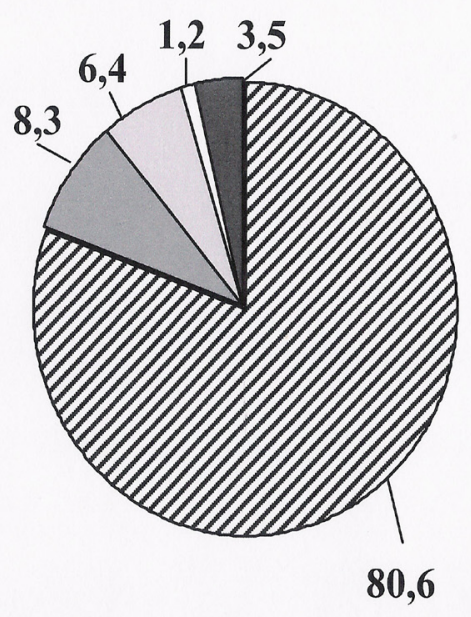

SS

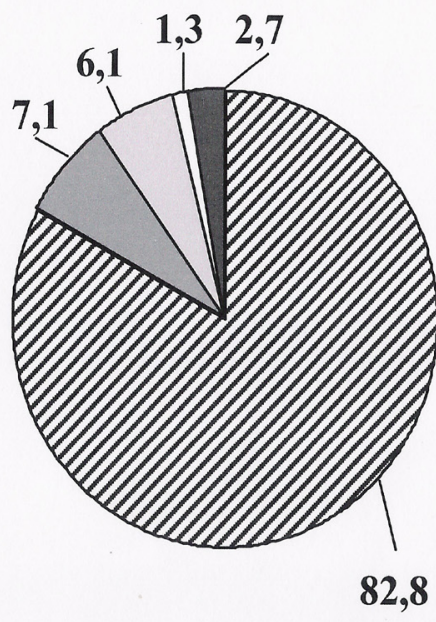

Total (CS + SS)

\section{Collembola}

\section{Acari $\square$ Hymenoptera}

\section{Coleoptera}

Outras

Figura 1. Distribuição geral dos principais grupos de artrópodes coletados em armadilha tipo alçapão no experimento com solarização (CS), sem solarização (SS) e total dos dois experimentos (CS+SS) (general distribution of main arthropods groups collected in pitfalls in solarized (CS), unsolarized (SS) and both (CS+SS) experiments). Brasília, UnB, 2005.

Tabela 2. Número de espécies predominantes, dominantes, abundantes, comuns, dispersas e raras selecionadas através dos índices faunísticos nas três épocas e nos cinco tratamentos de adubação para os dois experimentos. (number of predominant, dominant, abundant, common, dispersed and rare species selected by faunistic index in solarizated and unsolarizated experiments in three periods and five fertilization treatments). Brasília, UnB, 2005.

\begin{tabular}{|c|c|c|c|c|c|c|}
\hline \multirow{2}{*}{ Épocas/adubações } & \multicolumn{5}{|c|}{ Índices faunísticos } & \multirow[b]{2}{*}{ Raras } \\
\hline & Predominantes & Dominantes & Abundantes & Comuns & Dispersas & \\
\hline \multicolumn{7}{|c|}{ Com solarização } \\
\hline 1 & 9 & 16 & 9 & 11 & 7 & 25 \\
\hline 2 & 14 & 32 & 14 & 14 & 4 & 58 \\
\hline 3 & 17 & 27 & 17 & 14 & 3 & 72 \\
\hline Orgânica & 10 & 34 & 15 & 19 & 5 & 52 \\
\hline $\mathrm{N}-\mathrm{NH}_{4}$ & 9 & 22 & 10 & 16 & 1 & 48 \\
\hline NPK & 9 & 21 & 11 & 15 & 10 & 34 \\
\hline Org. + NPK & 12 & 26 & 18 & 15 & 12 & 56 \\
\hline Testemunha & 10 & 25 & 15 & 12 & 0 & 51 \\
\hline \multicolumn{7}{|c|}{ Sem solarização } \\
\hline 1 & 9 & 14 & 9 & 8 & 12 & 15 \\
\hline 2 & 14 & 37 & 14 & 20 & 12 & 59 \\
\hline 3 & 16 & 35 & 16 & 11 & 12 & 73 \\
\hline Orgânica & 10 & 30 & 16 & 16 & 12 & 58 \\
\hline $\mathrm{N}-\mathrm{NH}_{4}$ & 8 & 24 & 14 & 21 & 7 & 45 \\
\hline NPK & 7 & 19 & 13 & 15 & 6 & 39 \\
\hline Org. + NPK & 6 & 35 & 8 & 27 & 29 & 42 \\
\hline Testemunha & 12 & 24 & 14 & 15 & 5 & 52 \\
\hline
\end{tabular}




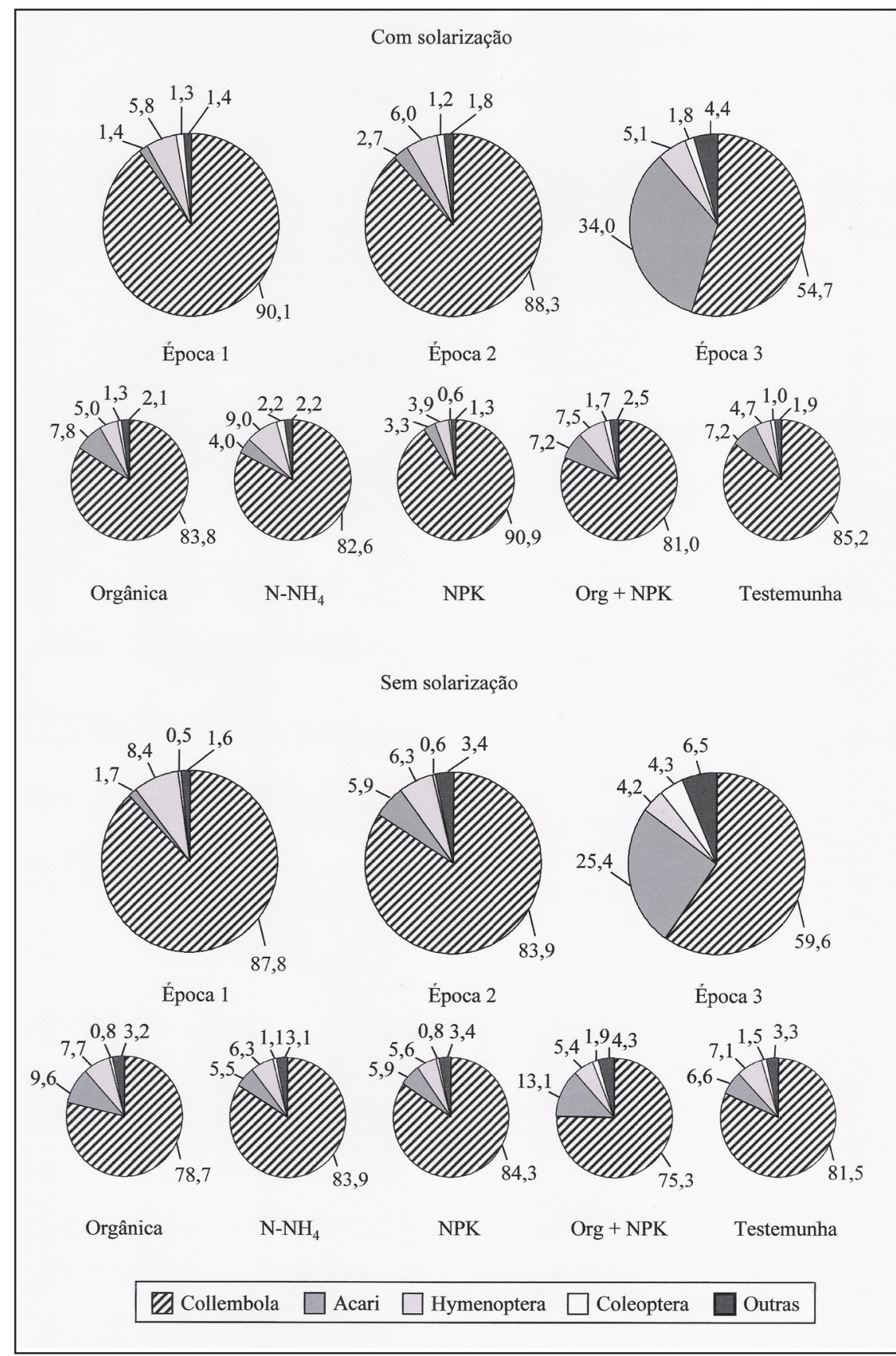

Figura 2. Distribuição percentual dos principais grupos de artrópodes coletados em tipo alçapão em cada uma das três épocas e tratamentos de adubação nos experimentos com solarização e sem solarização (percentual distribution of main arthropods groups collected in pitfalls in three epochs and five fertilization treatments in solarized and unsolarized experiments). Brasília, UnB, 2005.

também se destacaram: Desoria trispinata (MacGillivray) (Collembola: Isotomidae), Conomyrma sp. (Insecta: Hymenoptera), Seira sp. (Collembola: Entomodrydae), Prostigmata sp. (Acari: Prostigmata), Pheidole sp. (Insecta: Hymenoptera) e Proisotoma minuta (Tullberg) (Collembola: Isotomidae).

No experimento com solarização foram coletados 9.375 espécimes dis- faunísticos, observou-se que os dois experimentos não foram significativamente diferentes (Tabela 1 e 2). Os índices de diversidade, riqueza de espécies e equitabilidade não foram significativamente diferentes nos dois experimentos (Tabela 1). A equitabilidade foi baixa nos dois experimentos $(\mathrm{E}=0,34 \mathrm{com}$ solarização e 0,36 sem solarização). Isso ocorre quando algumas espécies apresentam número de indivíduos muito maior que as demais (Frizzas, 2003), o que pode ser confirmado pela diferença observada no número de espécies abundantes e raras, onde, cerca de $70 \%$ das espécies coletadas foram raras.

Observou-se que nos dois experimentos a época 2 apresentou valores significativamente maiores para o total coletado, seguidos pelas épocas 1 e 3 , respectivamente e que o número de espécies foi significativamente maior nas épocas 2 e 3 (Tabela 1).

A época 3 apresentou os menores valores para o total de espécimes coletados, e os maiores valores para o total de espécies. Isso condiz com os maiores índices de equitabilidade observados para essa época ( $E=0,74$ e 0,69$)$ (Tabela 1$)$. Considerando que fatores físicos como temperatura e umidade do solo afetam a comunidade de artrópodes (Oliveira et al., 2006; Doblas-Miranda et al., 2007), é possível que antes da implantação da cultura, quando não havia vegetação nas áreas experimentais, a comunidade de artrópodes apresentava um equilíbrio que mudou com a presença da cultura, a qual alterou o microclima do solo, por fatores como sombreamento, irrigação e presença das raízes. Essas novas características das áreas experimentais podem ter propiciado condições microclimáticas mais favoráveis ao estabelecimento de um número maior de espécies, e por competição interespecífica uma redução no número total de indivíduos.

Comparando-se cada uma das épocas entre os dois experimentos não se observaram diferenças para os índices de riqueza, diversidade e equitabilidade. Entretanto, entre as épocas dentro de cada experimento esses índices aumentaram da época 1 para a época 3 (Tabela 1). O índice de diversidade de Shannon-Wiener é bastante útil para uso em comparações de comunidade, 
Tabela 3. Total de espécimes coletados, número de espécies, riqueza, diversidade e equitabilidade em alçapão nos experimentos com solarização e sem solarização para os cinco tratamentos de adubação em cada época (total of collected specimens, number of species, richness index, diversity index and equitability index in pitfalls in solarizated and unsolarizated experiments in five fertilizations treatments in each epoch). Brasília, UnB, 2005.

\begin{tabular}{|c|c|c|c|c|c|c|}
\hline Tratamento & Época & Total coletado & $\begin{array}{c}\mathrm{N}^{\circ} \text { de } \\
\text { espécies }\end{array}$ & $\begin{array}{c}\text { Riqueza } \\
(\alpha)\end{array}$ & $\begin{array}{c}\text { Diversidade } \\
\left(\mathrm{H}^{\prime}\right) \\
\end{array}$ & $\begin{array}{c}\text { Equitabilidade } \\
\text { (E) }\end{array}$ \\
\hline \multicolumn{7}{|c|}{ Com solarização } \\
\hline \multirow{3}{*}{ Orgânica } & 1 & $619 a$ & $31 \mathrm{a}$ & $4,67 \mathrm{a}$ & $1,67 \mathrm{a}$ & $0,49 \mathrm{a}$ \\
\hline & 2 & $1192 a$ & $46 \mathrm{a}$ & $6,35 \mathrm{a}$ & $1,76 \mathrm{a}$ & $0,46 \mathrm{a}$ \\
\hline & 3 & $334 \mathrm{a}$ & $49 \mathrm{a}$ & $8,26 \mathrm{a}$ & $2,89 \mathrm{a}$ & $0,74 \mathrm{a}$ \\
\hline & 1 & $453 b$ & $23 \mathrm{a}$ & $3,60 \mathrm{a}$ & $1,63 \mathrm{~b}$ & $0,52 b$ \\
\hline \multirow[t]{2}{*}{$\mathrm{N}-\mathrm{NH}_{4}$} & 2 & $1056 \mathrm{a}$ & $43 \mathrm{a}$ & $6,03 \mathrm{a}$ & $1,96 a b$ & $0,52 b$ \\
\hline & 3 & $113 \mathrm{c}$ & $43 \mathrm{a}$ & $8,88 \mathrm{a}$ & $3,37 \mathrm{a}$ & $0,90 \mathrm{a}$ \\
\hline & 1 & $670 \mathrm{a}$ & $17 \mathrm{a}$ & $2,46 b$ & $1,41 \mathrm{~b}$ & $0,50 \mathrm{~b}$ \\
\hline \multirow[t]{2}{*}{ NPK } & 2 & $1273 a$ & $42 \mathrm{a}$ & $5,74 \mathrm{ab}$ & $1,68 b$ & $0,45 b$ \\
\hline & 3 & $150 \mathrm{a}$ & $39 \mathrm{a}$ & $7,58 \mathrm{a}$ & $3,14 \mathrm{a}$ & $0,86 \mathrm{a}$ \\
\hline \multirow{3}{*}{ Orgânica + NPK } & 1 & $648 \mathrm{a}$ & $26 a$ & $3,86 \mathrm{~b}$ & $1,45 b$ & $0,45 \mathrm{ab}$ \\
\hline & 2 & $686 a$ & $48 \mathrm{a}$ & 7,20ab & $2,15 b$ & $0,56 \mathrm{~b}$ \\
\hline & 3 & $275 \mathrm{a}$ & $66 \mathrm{a}$ & $11,57 \mathrm{a}$ & $3,43 \mathrm{a}$ & $0,82 \mathrm{a}$ \\
\hline \multirow{3}{*}{ Testemunha } & 1 & $574 a$ & $20 \mathrm{~b}$ & $2,99 \mathrm{~b}$ & $1,48 \mathrm{~b}$ & $0,49 a$ \\
\hline & 2 & $1115 \mathrm{a}$ & $45 \mathrm{a}$ & $6,27 \mathrm{a}$ & $1,92 \mathrm{ab}$ & $0,50 \mathrm{a}$ \\
\hline & 3 & $217 \mathrm{a}$ & $46 \mathrm{a}$ & $8,36 \mathrm{a}$ & $2,90 \mathrm{a}$ & $0,76 \mathrm{a}$ \\
\hline \multicolumn{7}{|c|}{ Sem solarização } \\
\hline \multirow{3}{*}{ Orgânica } & 1 & $544 a$ & $21 \mathrm{~b}$ & $3,18 b$ & $1,50 \mathrm{~b}$ & $0,49 b$ \\
\hline & 2 & $1308 \mathrm{a}$ & $63 a$ & $8,64 \mathrm{a}$ & $2,10 \mathrm{a}$ & $0,51 b$ \\
\hline & 3 & $266 \mathrm{a}$ & $55 \mathrm{ab}$ & $9,67 \mathrm{a}$ & $3,19 \mathrm{a}$ & $0,80 \mathrm{a}$ \\
\hline \multirow{3}{*}{$\mathrm{N}-\mathrm{NH}_{4}$} & 1 & $579 a$ & $24 a$ & $3,62 \mathrm{a}$ & $1,48 \mathrm{a}$ & $0,46 b$ \\
\hline & 2 & $1073 a$ & $46 a$ & $6,45 \mathrm{a}$ & $1,84 \mathrm{a}$ & $0,48 b$ \\
\hline & 3 & $309 \mathrm{a}$ & $47 \mathrm{a}$ & $8,02 \mathrm{a}$ & $3,00 \mathrm{a}$ & $0,78 \mathrm{a}$ \\
\hline & 1 & $538 \mathrm{a}$ & $25 \mathrm{a}$ & $3,82 \mathrm{a}$ & $1,67 \mathrm{a}$ & $0,54 a$ \\
\hline \multirow[t]{2}{*}{ NPK } & 2 & $857 \mathrm{a}$ & $31 \mathrm{a}$ & $4,44 \mathrm{a}$ & $1,64 \mathrm{a}$ & $0,48 \mathrm{a}$ \\
\hline & 3 & $326 \mathrm{a}$ & $48 \mathrm{a}$ & $8,12 \mathrm{a}$ & $2,87 \mathrm{a}$ & $0,74 \mathrm{a}$ \\
\hline & 1 & $484 \mathrm{a}$ & $20 \mathrm{~b}$ & $3,07 \mathrm{a}$ & $1,65 \mathrm{a}$ & $0,55 \mathrm{a}$ \\
\hline \multirow[t]{3}{*}{ Orgânica + NPK } & 2 & $962 \mathrm{a}$ & $63 a$ & $9,03 \mathrm{a}$ & $2,38 \mathrm{a}$ & $0,57 \mathrm{a}$ \\
\hline & 3 & $589 \mathrm{a}$ & $61 \mathrm{a}$ & $9,41 \mathrm{a}$ & $2,55 \mathrm{a}$ & $0,62 \mathrm{a}$ \\
\hline & 1 & $330 \mathrm{a}$ & $24 \mathrm{a}$ & $3,97 \mathrm{a}$ & $1,79 \mathrm{a}$ & $0,56 a b$ \\
\hline \multirow[t]{2}{*}{ Testemunha } & 2 & $1167 \mathrm{a}$ & $51 \mathrm{a}$ & $7,08 \mathrm{a}$ & $1,83 \mathrm{a}$ & $0,47 \mathrm{~b}$ \\
\hline & 3 & $214 \mathrm{a}$ & $46 \mathrm{a}$ & $8,39 \mathrm{a}$ & $2,93 \mathrm{a}$ & $0,77 \mathrm{a}$ \\
\hline
\end{tabular}

Médias de épocas seguidas de mesma letra minúscula dentro de cada adubação, em cada experimento, não diferem pelo intervalo de confiança do teste $\mathrm{t}$ a 0,05 de probabilidade. (Periods averages folowed by same small letter in each fertilization treatment in each experiment did not differ from each other by the t test at $5 \%$ probability).

pois, considera o número de espécies amostradas (riqueza) e a sua representação proporcional na amostra (Frizzas, 2003).

Comunidades com maiores índices de diversidade tendem a ser mais estáveis, uma vez que espécies raras aparentemente sem importância podem ser fundamentais para a manutenção da biodiversidade (Santos \& Marques 1996). No entanto, baixos índices de diversidade são esperados em ambientes agrícolas (Frizzas, 2003). Os fatores que contribuíram para os aumentos nos índices de riqueza, diversidade e equitabilidade, observados da época 1 para época 3 podem estar relacionados aos distúrbios causados pelo preparo do solo pouco antes da época 1 , desfavorecendo algumas espécies de artrópodes (Oliveira et al., 2006).

A equitabilidade foi significativamente menor nas épocas 1 e 2 nos dois experimentos, ou seja, baixa uniformidade na distribuição das espécies, $o$ que pode ser explicado pela dominância dos colêmbolos em relação aos demais 
grupos (Figura 2). Entretanto, valores de equitabilidade significativamente mais elevados foram observados na época 3 para os dois experimentos, o que pode ser atribuído ao maior número de espécies coletadas e também ao aumento da população de ácaros e de espécies raras (Tabelas 1 e 2).

Os dois experimentos foram semelhantes quanto à distribuição dos principais grupos de artrópodes (Figura 2), predominando nas épocas 1 e 2 os colêmbolos, representando mais de $80 \%$ do total de artrópodes coletado.

$\mathrm{Na}$ época 3, embora os colêmbolos ainda permanecessem em maior número, a porcentagem de ácaros aumentou substancialmente, passando de menos de $6 \%$ para $25,4 \%$ e $34 \%$ nos experimentos sem e com solarização, respectivamente. Assim, a presença da cultura parece ter influenciado mais a comunidade de artrópodes do que a solarização, sugerindo que a qualidade do solo não foi negativamente alterada pela solarização.

A população de ácaros pode ser considerada um indicador de impacto, pois tende a diminuir com aumento da intensidade de cultivo e uso de pesticidas, dentre outros fatores (Ferraro et al., 2007), o que não ocorreu em nenhum dos dois experimentos.

A população de oribatídeos aumentou da época 1 para a época $3\left(\mathrm{E}_{1}=\right.$ $0 ; \mathrm{E}_{2}=0 ; \mathrm{E}_{3}=6$ no experimento com solarização e $\mathrm{E}_{1}=0 ; \mathrm{E}_{2}=0 ; \mathrm{E}_{3}=5$ no experimento sem solarização), o que reforça a hipótese de que a solarização não afetou a população de artrópodes, já que a população desses ácaros tendem a diminuir em condições desfavoráveis (Duarte, 2004). No presente estudo, os tratamentos de adubação podem ter contribuído para aumento da população de ácaros na época 3, conforme relatado por Leroy et al. (2007).

Nos dois experimentos, as espécies que mais se destacaram na época 1 foram P. tenella; S. atrolutea e F. centralis, sendo que as duas primeiras representaram cerca de $70 \%$ do total coletado.

$\mathrm{Na}$ época 2, no experimento com solarização, destacou-se a espécie $S$. atrolutea, representando 50\%, e, no experimento sem solarização, destacou-se P. tenella, com $38 \%$ do total.
A espécie $S$. atrolutea foi aparentemente favorecida pela solarização enquanto no experimento sem solarização a espécie $P$. tenella predominou.

$\mathrm{Na}$ época 3 também se observou diferença entre os experimentos. No experimento com solarização destacaramse as espécies $P$. tenella; Isotomurus sp., sendo esta última não coletada nas duas épocas anteriores, e a espécie de ácaro Rosensteniidae sp. 86 que esteve presente apenas na época 2 e em pequeno número. No experimento sem solarização destacaram-se as espécies de Collembola Desoria trispinata (MacGillivray) e $P$. tenella e a espécie de ácaro Prostigmata sp. 202.

A primeira não esteve presente nas épocas 1 e 2 e a última foi coletada na época 2, mas em pequena quantidade. Observou-se que nos dois experimentos, na época 3, as espécies que se destacaram representavam menos de $50 \%$ do total coletado, provavelmente em função da maior diversidade de espécies observadas nessa época.

Alguns ácaros prostigmatas são predadores de artrópodes que vivem no solo e de seus ovos (Spain \& Hutson, 1983). Na época 3, as espécies de prostigmatas aumentaram suas populações, provavelmente favorecidas por algumas outras espécies de artrópodes que lhe serviram como alimento, e que possivelmente não estavam presentes nas épocas anteriores.

O total de espécimes coletados e o número de espécies coletadas não foram afetados pelos tratamentos de adubação dentro de cada um dos experimentos, considerando-se as três épocas avaliadas (Tabela 1). Comparando-se os dois experimentos, observou-se comportamento semelhante, tendo o mesmo ocorrido para os índices de riqueza, diversidade e equitabilidade (Tabela 1).

Embora os valores de riqueza de espécies $(\alpha=13,78)$ e diversidade $\left(H^{\prime}=\right.$ $2,77)$ tenham sido mais elevados em termos absolutos para o tratamento de adubação orgânica + NPK no experimento sem solarização (Tabela 1), eles não diferiram significativamente dos demais tratamentos.

Quanto à equitabilidade, observouse baixa uniformidade na distribuição das espécies (valores próximos de 0,50 em todos os tratamentos). Isso demonstra que algumas espécies apresentaram um número de indivíduos coletados muito maior que as demais, ou seja, muitas espécies coletadas foram raras e poucas foram abundantes em todos os tratamentos nos dois experimentos (Tabela 2).

As espécies que mais se destacaram em todos os tratamentos de adubação no experimento com solarização, em ordem decrescente de abundância, foram $S$. atrolutea, $P$. tenella e $F$. centralis, que juntas representaram cerca de $70 \%$ do total coletado. No experimento sem solarização as espécies que mais se destacaram em todos os tratamentos, em ordem decrescente de abundância foram P. tenella; S. atrolutea e F. centralis, também representando cerca de $70 \%$ do total coletado.

Quanto à distribuição dos principais grupos de artrópodes coletados, observou-se semelhança entre os tratamentos de adubação (Figura 2).

Em todos os tratamentos a classe Collembola foi predominante. No tratamento com NPK no experimento com solarização, essa classe representou $90,9 \%$ do total coletado enquanto no tratamento de adubação orgânica + NPK a porcentagem de colêmbolos coletados diminuiu para $81 \%$, sendo a população de ácaros maior em relação aos outros tratamentos. Estudos realizados em uma floresta mista, a quantidade e a composição da matéria orgânica foi a variável que mais influenciou na organização da comunidade de artrópodes do solo (Hasegawa, 2001).

Os colêmbolos se alimentam principalmente de microrganismos e fungos associados à matéria orgânica do solo (Spain \& Hutson, 1983; Zeppelini Filho \& Belini, 2004), e aqueles pertencentes à família Entomodrydae (Greensland \& Greensland, 1983), por exemplo, à qual pertencem também várias espécies que foram predominantes no presente estudo, dentre elas $S$. atrolutea (Tabelas 1 e 2) se alimentam principalmente de fungos, pólen e algas.

Espécies pertencentes à família Isotomidae, à qual pertencem $P$. tenella, F. centralis, Isotomurus sp. e D. trispinata dentre outras, se alimentam de material vegetal em decomposição e 
em menor proporção de hifas fúngicas e esporos (Greensland \& Greensland, 1983). Considerando que o teor de matéria orgânica no solo dos experimentos avaliados foi de médio a bom, variando de 37,9 a 42,6 $\mathrm{g} \mathrm{kg}^{-1}$, essa pode ser uma explicação para o fato dos colêmbolos terem representado a maior proporção dos artrópodes coletados.

O teor de matéria orgânica do solo ou mesmo a adição de fertilizantes orgânicos pode favorecer a comunidade de artrópodes, sobretudo colêmbolos e ácaros (Leroy et al., 2007; Ferraro et al., 2007).

Com relação às épocas, na maioria dos tratamentos de adubação, nos dois experimentos, o total de espécimes coletados foi maior na época 2 embora os valores não difiram, com exceção de $\mathrm{N}-\mathrm{NH}_{4}$ (Tabela 3). Quanto ao número de espécies, no experimento solarizado, com exceção da testemunha em que o número de espécies foi significativamente maior nas épocas 2 e 3, nos demais tratamentos não houve diferença entre épocas. Já no experimento sem solarização, os tratamentos de adubação orgânica e orgânica + NPK apresentaram aumento do número de espécies nas épocas 2 e 3 (Tabela 3 )

Os índices de riqueza, diversidade e equitabilidade apresentaram tendência semelhante nos dois experimentos, com aumento da época 1 para a época 3 (Tabela 3). Ressalta-se que a equitabilidade foi substancialmente maior na época 3 para todos os tratamentos, nos dois experimentos, sendo maior apenas nos tratamentos $\mathrm{N}_{-} \mathrm{NH}_{4}$, NPK e orgânica + NPK no experimento solarizado e orgânica, $\mathrm{N}_{-} \mathrm{NH}_{4}$ e testemunha no experimento não solarizado (Tabela 3 ). Isso sugere que a presença da cultura na época 3 afetou mais a comunidade de artrópodes do que os tratamentos de adubação aumentando a diversidade de espécies. Conclui-se, portanto, que a solarização associada à adubação não afetou a comunidade de artrópodes e o seu uso não apresenta restrição quanto à qualidade do solo.

\section{AGRADECIMENTOS}

À CAPES pela concessão de bolsa à primeira autora; à $\operatorname{Dr}^{\mathrm{a}}$ Maria Cleide de Mendonça (Museu Nacional/UFRJ), Dr $^{\mathrm{a}}$ Marina Regina Frizzas (UniCEUB), Dr.Aníbal R. Oliveira (ESALQ/USP), $D^{\mathrm{a}}{ }^{\mathrm{a}}$ Denise Navia Magalhães Ferreira (Embrapa Cenargen), Dr. Sérgio Antônio Vanin (Instituto Biológico de São Paulo), Dr. Carlos Campaner (Museu de Zoologia da USP), Dr ${ }^{\text {a }}$. Helena Castanheira de Morais, Dr. Paulo César Motta, Dr. José Roberto Pujol Luz, Dr. Reginaldo Constantino (UnB) pelo auxílio na identificação taxonômica de parte do material coletado.

\section{REFERÊNCIAS}

ASSAD MLL. 1997. Fauna do solo. In: VARGAS MAT; HUNGRIA M. Biologia dos solos dos cerrados. Planaltina: Embrapa CPAC. p.363-443.

BADJI CA; GUEDES RNC; SILVAAA; CORRÊA AS; QUEIROZ MELR; MICHERELF-FILHO M. 2007. Non-target of deltamethrin on soil arthropods of maize fields under conventional and no-tillage cultivation. Journal of Applied Entomology 131: 50-58.

DOBLAS-MIRANDA E; SÁNCHEZ-PIÑERO F; GONZÁLEZ-MEGÍAS A. 2007. Soil macroinvertebrate fauna of a Mediterranean arid system: Composition and temporal changes in the assemblage. Soil Biology \& Biochemistry 39: 1916-1925.

DUARTE MM. 2004. Abundância de microartrópodes do solo em fragmentos de mata com araucária no sul do Brasil. Iheringia, Série Zoologia 94: 163-169.

ECKERT J; SCHUPHAN I; HOTHORN LA; GATHMANN A. 2006. Arthropods on maize ears for detecting impacts of $\mathrm{Bt}$ maize on nontarget organisms. Environmental Entomology 35: 554-560.

FERRARO D; GHERSA CM. 2007. Exploring the natural and human-induced effects on the assemblage of soil microarthropod communities in Argentina. European Journal of Soil Biology 43: 109-119.

FRIZZAS MR. 2003. Efeito do milho geneticamente modificado MON810 sobre a comunidade de insetos. Piracicaba: USP ESALQ. 192p (Tese doutorado).

GHINI R; PATRICIO FRA; SOUZA MD; SINIGAGLIA C; BARROS BC; LOPES MEBM; TESSARIOLI NETO J; CANTARELLAH. 2003. Efeito da solarização sobre propriedades físicas, químicas e biológicas de solos. Revista Brasileira de Ciência do Solo 27: 71-79.

GREENSLADE PJM; GREENSLADE. 1983. Ecology of soil invertebrates. In: Soils: an Australian viewpoint. Division of Soils, CSIRO pp. 645-669 (CSIRO: Melbourne/ Academic Press: London).

HASEGAWA M. 2001. The relationship between the organic matter composition of a forest floor and the structure of a soil arthropod community. European Journal of Soil Biology 37: 281-284.

HASING JE; MOTSENBOCKER CE; MONLEZUN CJ. 2004. Agroeconomic effect of soil solarization on fall-planted lettuce (Lactuca sativa). Scientia horticulturae 101: 223-233.

KOUNDA-KIKI C; VAÇULIK A; PONGE JF; SARTHOU C. 2004. Soil arthropods in a development succession on the Nouragues inselbergs (French Guiana). Biology and Fertility of Soils 40: 119-127.

LEROY BLMM; BOMMELE L; REHEUL D; MOENS M; NEVE E. 2007. The application of vegetable, fruit and garden waste (VFG) compost in addition to cattle slurry in a silage maize monoculture: Effects on soil fauna and yield. European Journal of Soil Biology 43: 91-100.

LINS VS; SANTOS HR; GONÇALVES MC. 2007. The effect of glyphosate, 2,4D, atrazine and nicosulfuron herbicides upon the edaphic Collembola (Artropoda:Ellipura) in a no tillage system. Neotropical Entomology 36:261267.

NAKAMURAA; CATTERALL C; HOUSE APN; KITCHING RL; BURWELL CJ. 2007. The use of ants and other soil and litter arthropods as bio-indicators of the impacts of rainforest clearing and subsequent land use. Journal of Insect Conservation 11:177-186.

OLIVEIRA CM; RESCK DVS; ICUMA IM; ALVES RT. 2002. Efeito do sistema de preparo do solo e rotação de culturas na dinâmica de população de artrópodes do solo. Planaltina: Embrapa Cerrados. Comunicado Técnico, 73.

OLIVEIRA MAS; RESCK DVS; FRIZZAS MR. 2006. Artrópodes edáficos: influência dos sistemas de preparo do solo e de rotação de culturas. Planaltina: Embrapa Cerrados. Boletim de Pesquisa e Desenvolvimento, 160.

PIMENTEL-GOMES F. 2000. Curso de estatística experimental. São Paulo: Nobel. 477p.

ROSE R; DIVELY GP. 2007. Effects of insecticidetreated and lepidopteran-active bt transgenic sweet corn on the abundance and diversity of arthropods. Environmental Entomology 36: 1254-1268.

SANTOS GMM; MARQUES OM. 1996. Analise faunística e comunidades de formigas epigéias (Hymenoptera, Formicidae) em dois ecossistemas em Cruz das Almas-BA. Insecta 5: 1-17.

SPAIN AV; HUTSON BR. 1983 Dynamics and fauna of the litter layers. In: Soils: an Australian viewpoint. Division of Soils, CSIRO pp.611-628 (CSIRO: Melbourne/ Academic Press: London).

WIWATWITAYA D; TAKEDA H. 2005. Seasonal changes in soil arthropod abundance in the dry evergreen forest of north-east Thailand, with special reference of collembolan communities. Ecological Research 20: 59-70.

ZEPPELINI FILHO D; BELLINI BC. 2004. Introdução ao estudo dos Collembola. João Pessoa: Editora UFPB. 82 p. 